\title{
MEHRSPRACHIGKEIT ALS RESSOURCE IM FREMDSPRACHENUNTERRICHT
}

\author{
Dr. Diana Babušytè \\ Mykolas Romeris Universität
}

\begin{abstract}
Annotation. Im Mittelpunkt des vorliegenden Beitrags steht die Frage der Förderung der individuellen Mehrsprachigkeit im Fremdsprachenunterricht. Hier werden die wichtigsten Prinzipien der Interkomprehensionsforschung präsentiert sowie bewährte EuroCom-Projekte behandelt, die Ähnlichkeiten zwischen verwandten Sprachen aufgreifen und auf die Förderung der rezeptiven Mehrsprachigkeit abzielen. Darüber hinaus wird das Instrument der sieben Siebe des EuroComGerm-Projekts vorgestellt. Es wird der Frage nachgegangen, wie die Erkenntnisse der Mehrsprachigkeitsdidaktik effizient als Ressource im Fremdsprachenunterricht eingesetzt werden und zur Förderung einer mehrsprachigen Kompetenz beitragen können.
\end{abstract}

Schlüsselwörter: Mehrsprachigkeit, Mehrsprachigkeitsdidaktik, Inter- und Eurokomprehension, EuroComGerm, Deutsch nach Englisch

\section{Einleitung}

Mehrsprachigkeit ist ein komplexer Begriff, der nicht einheitlich aufgefasst wird (Surkamp, 2010: 214). Einerseits unterscheidet man die so genannte individuelle Mehrsprachigkeit, d.h. die Fähigkeit eines Menschen, sich mehr als einer Sprache zu bedienen (Multilingualismus, Plurilingualismus); andererseits kann Mehrsprachigkeit die Verwendung mehrerer Sprachen innerhalb einer Gesellschaft, eines Sprachgebiets, eines Staates bezeichnen (gesellschaftliche Vielsprachigkeit, Polyglossie). Die Europäische Kommission veröffentlichte im Jahre 2005 folgende Definition: „Unter Mehrsprachigkeit versteht man sowohl die Fähigkeit einer Person, mehrere Sprachen zu benutzen, als auch die Koexistenz verschiedener Gemeinschaften in einem geographischen Raum." (Kommission der europäischen Gemeinschaften, 2005: 596).

Das Phänomen der Mehrsprachigkeit spielt in der Europäischen Union eine wichtige Rolle. Europa wächst als wirtschaftliche und politische Einheit zusammen, deswegen ist es für die gegenseitige Verständigung von Vorteil, dass wir Europäer mehr Fremdsprachen lernen (Neuner, 2004: 38). Dementsprechend lautet die Empfehlung der EU: Jeder Bürger Europas sollte neben seiner Muttersprache zwei 
weitere europäische Sprachen beherrschen. Weiterhin wird unsere moderne Gesellschaft im Zuge der steigenden professionellen Mobilität und der gegenwärtigen globalen Migration vor neue Herausforderungen gestellt. In diesem Kontext kommt dem Fremdsprachenlernen eine große Bedeutung zu (Cosentino, 2016: 90).

Die Mehrsprachigkeitsdidaktik, ein relativ junger Begriff in der europäischen und weltweiten Sprachendidaktik, beruht darauf, das Interesse der Lerner an Fremdsprachen und Fremdsprachenlernen zu entwickeln sowie eine Distanz zum traditionellen monolingualen Konzept im Fremdsprachenunterricht zu schaffen. Sie stellt den Lernenden ins Zentrum ihres Interesses und ist auf den Ausbau individueller Mehrsprachigkeit ausgerichtet.

Den Untersuchungsgegenstand des vorliegenden Beitrags bilden die Ansätze der Mehrsprachigkeitsdidaktik im Fremdsprachenunterricht. Der Beitrag setzt sich zum Ziel, die Methode EuroComGerm in ihrer Bedeutung für die Förderung der individuellen Mehrsprachigkeit im Rahmen des Fremdsprachenunterrichts vorzustellen. Für die Verwirklichung dieses Ziels sind folgende Aufgaben zu erfüllen: Es werden Inter- und Eurokomprehension dargestellt und deren fundamentale Prinzipien beschrieben. Darüber hinaus werden die „sieben Siebe“ der EuroComGerm-Methode als Lernstrategien erläutert, die auf dem kontrastiven Vergleich von Sprachen auf der Grundlage der Lesekompetenz basieren. Dabei wird die deskriptiv-analytische Methode verwendet.

\section{Inter- und Eurokomprehension}

Als Teil der Mehrsprachigkeitsdidaktik findet Interkomprehension ihren Ursprung in den 1990er Jahren. Sie hebt den Einbezug verschiedener Sprachen im Fremdsprachenlernprozess hervor und richtet sich auf die Kommunikation zwischen Mitgliedern einer Sprachfamilie. Dabei setzt sie sich zum Ziel, durch Ähnlichkeiten zwischen verwandten Sprachen, eine Lese- und Verstehenskompetenz zu erreichen (Perge, 2014: 266).

$\mathrm{Zu}$ den fundamentalen Prinzipien der Interkomprehension gehören folgende Schritte (Bär, 2012: 14):

$\square$ das Prinzip der Bewusstmachung (kognitives Lernen)

$\square$ das Prinzip der Lernerautonomisierung (entdeckendes Lernen)

$\square$ das Prinzip der Authentizität (inhaltsorientiertes Lernen)

$\square$ das Prinzip der Rezeptivität (verstehensorientiertes Lernen)

Das Prinzip des kognitiven Lernens verhilft dem Lerner, seinen Lernprozess bewusst zu entwickeln. Die Sprache wird im Unterricht aktiv entdeckt und mit anderen Sprachen verglichen. Dabei werden Interferenz- und Transferstrukturen identifiziert, die zur Erleichterung des Lernprozesses beitragen.

Das Prinzip der Lernerautonomisierung erzielt eine eigenständige Erforschung der unbekannten Sprachen durch Vergleiche und die Analyse des eigenen Lernprozesses. Außer der Selbstreflexion und der Eigenständigkeit der Lerner ge- 
hört zu diesem Prinzip auch die Vermittlung verschiedener Lernstrategien, wie beispielsweise die „sieben Siebe“ der EuroCom-Methode, die im nächsten Abschnitt detailliert präsentiert werden.

Das Prinzip der Authentizität beruht auf dem Einsatz authentischer Lehrmaterialien im Unterricht, die die Interessen der Lerner berücksichtigen. Dieses Priznip zeichnet sich in seiner Echtheit aus, indem die Sprache in ihrer Realität kennen gelernt wird.

Das Prinzip der Rezeptivität ist grundlegend für das interkomprehensive Lernen, denn sein Ziel ist das sinngemäße Erschließen und Verstehen der neuen Zielsprache (Bär, 2012: 20).

Der Begriff „Eurokomprehension“ entstand in Anlehnung an die europäische Interkomprehension. „Die europäische Intercomprehensionsforschung hat zum Ziel, in einer Gruppe von Sprachen, die einen gemeinsamen Ursprung haben, kommunizieren zu können, wobei sich jeder seiner Muttersprache bedient." (Klein 2001)

Das Projekt EuroCom stellt einen Ansatz dar, die Mehrsprachigkeit innerhalb der Sprachfamilien zu nutzen, um die autochtone Kommunikation als Alternative zum Englischen als einer einzigen Lingua franca zu stärken und die Kulturräume Europas zu stützen. Es wurde Ende der 90er Jahre unter der Leitung des Romanisten Horst G. Klein an der Universität Frankfurt am Main entwickelt. Es geht hier um eine Art „Didaktische Linguistik“, deren Ziel es ist, eine rezeptive Mehrsprachigkeit zu einem europaweit einsetzbaren Programm zu gestalten. Das Englische sollte dabei auf dem Gebiet einer Sprachfamilie zurückgedrängt werden. Dem Projekt liegt folgende Idee zugrunde: Man spricht Sprache A, hört und versteht Sprache B. Das hat sich auch in der Praxis bewährt, z. B. auf Kongressen, so wird die Unterprivilegierung einer Seite vermieden (Hinrichs, 2009: 31-32).

EuroCom ist auf die großen Sprachfamilien konzentriert: die romanischen Sprachen (EuroComRom) (Klein 2001), die slawischen Sprachen (EuroComSlav) (Zybatow 2002), die germanischen Sprachen (EuroComGerm) (Hufeisen, Marx 2007, 2014). Um von der Erstsprache (meistens Französisch, Deutsch, Russisch) zur Zweitsprache zu gelangen (z. B. Rumänisch, Niederländisch, Bulgarisch), werden im Lernprogramm verschiedene Sprachfelder benutzt (die sog. „Transferbasen"). Sie beschleunigen das Satzverstehen, indem sie das Gemeinsame bzw. Bekannte für beide Sprachen hervorheben. Sie stellen die so genannten „sieben Siebe“ dar und enthalten den internationalen Wortschatz, die Erbwörter, Lautentsprechungen, die Graphiksysteme, die flektierten Wortarten, das Wichtigste aus Syntax und Wortbildung (Hinrichs, 2009: 32).

\section{Das EuroCom Germ-Projekt und das Instrument der sieben Siebe}

Da die germanischen Sprachen zu den mittlerweile häufig gelernten Fremdsprachen an litauischen Hochschulen gehören, wird in diesem Abschnitt das EuroComGerm-Projekt bzw. die EuroComGerm-Methode detaillierter vorgestellt. 
EuroComGerm ermöglicht Sprechern germanischer Sprachen, Texte in weiteren germanischen Sprachen zu verstehen, indem sie viel Bekanntes entdecken und entschlüsseln können (Hufeisen, Marx, 2007: 4). Genauso wie EuroComRom und EuroComSlav stellt das EuroComGerm-Projekt das Lesen als rezeptive Fertigkeit in den Mittelpunkt. Dabei werden die zwischen den Sprachen bestehenden Ähnlichkeiten als Ressource bzw. als Grundlage beim Verstehen betrachtet (Hufeisen, Marx, 2007: 3).

Um den Gesamtsinn eines Textes in einer unbekannten Sprache erfassen zu können, werden beim Lesen verschiedene Strategien eingesetzt, die als ,sieben Siebe“ bezeichnet werden. „Jedes einzelne Sieb verhilft dem Lernenden einen Schritt weiter Richtung Textverständnis" (Hufeisen, Marx, 2014: 9). Das sind Strategien, die zum optimierten Texterschließen verwendet werden und die sieben Sprachbereiche als Transferbasen beim Erschließen darstellen (Hufeisen, Marx, 2007: 7). „Das heißt, dass man zunächst nur rezeptive Kompetenzen schriftlicher Texte anvisiert. Hierbei erzielt man keine, perfekten' Kenntnisse, sondern eignet sich vor allem Strategien an, wie an Texte aus verwandten Sprachen heranzugehen ist, um schnell zu einem globalen, und dank der Sprachverwandtschaft direkt zu einem detaillierten Verständnis, zu gelangen. " (Hufeisen, Marx, 2007: 5)

Die sieben Siebe im EuroComGerm-Ansatz sind folgende:

- Sieb 1: Internationalismen und gemeinsamer germanischer Wortschatz;

- Sieb 2: Funktionswörter;

- Sieb 3: Laut- und Graphementsprechungen;

- Sieb 4: Rechtschreibung und Aussprache;

- Sieb 5: Syntaktische Strukturen;

- Sieb 6: Morphosyntaktische Strukturen;

- Sieb 7: Prä- und Suffixe.

Im Weiteren werden die einzelnen ,sieben Siebe“ als Erschließungsstrategien genauer erläutert. Dabei beziehen sich die Beispiele hauptsächlich auf die zweite Auflage von Britta Hufeisen und Nicole Marx (2014), in der folgende Sprachen behandelt werden: Dänisch, Englisch, Friesisch, Isländisch, Niederländisch, Norwegisch (Bokmå//Nynorsk) und Schwedisch.

Das erste Sieb „Internationalismen und gemeinsamer germanischer Wortschatz" beinhaltet die meisten auffälligen Ähnlichkeiten, die den ersten Anhaltspunkt für den Textinhalt bieten. Der internationale Wortschatz drückt sich in den großen Entlehnungsbewegungen aus, die zur Entstehung und Verbreitung von Internationalismen geführt haben. Das sind Übernahmen aus dem Französischen, Italienischen, Englischen und Arabischen sowie aus kleineren Sprachen, die ihre Spuren weltweit hinterlassen haben. Sie beziehen sich größtenteils auf einheimische Lebensumstände, Gebräuche, landestypische Produkte und Gegenstände. Im Deutschen sind es beispielsweise bis zu 5000 Wörter (Hufeisen, Marx, 2014: 11): Französisch: Allee, Mode, charmant, Dessert, delikat, Mayonnaise, Kotelett, Kognak etc. Italienisch: Aquarell, Arie, netto, Pizza, Espresso, Mozzarella, Prosecco, Spaghetti etc. 
Englisch: Slogan, Manager, Start, Disko, Hit, Jazz, Show, Sex, Export, Import etc. Deutsch: der Walzer: finn. valssi, poln. walc, span. vals, kroat. valcer, lit. valsas etc. Arabisch: Alkohol, Jasmin, Kaffee, Zucker, Algebra, Havarie, Alchemie, Elixier etc. Japanisch: Sumo, Judo, Karate, Sushi, Karaoke, Kamikaze, Sake, Tsunami etc. Türkisch: Harem, Pascha, Kismet, Döner, Yoghurt etc.

Skandinavische Sprachen: Fjord, Saga, Geysir, Sauna etc.

Ein anderer Teil des gemeinsamen Wortschatzes gehört zum Erbwortschatz, dessen Übereinstimmungen innerhalb der germanischen Sprachen eine bessere Orientierung über den Textinhalt ermöglichen (Hufeisen, Marx, 2007: 8), z.B.:

\begin{tabular}{|c|c|c|c|c|c|c|}
\hline Schw. & Norw. & Dän. & Isl. & Engl. & Nl. & Dt. \\
\hline ja & ja & ja & já & yes & ja & ja \\
\hline nej & nei & nej & nei & no & nee(n) & nein \\
\hline dag & dag & dag & dagur & day & dag & Tag \\
\hline natt & natt & nat & nótt & night & nacht & Nacht \\
\hline land & land & land & land & land & land & Land \\
\hline sommar & sommer & sommer & sumar & summer & zomer & Sommer \\
\hline katt & katt & kat & köttur & cat & kat & Katze \\
\hline bok & bok & bog & bók & book & boek & Buch \\
\hline här & her & her & hér & here & hier & hier \\
\hline son & sønn & søn & sonur & son & zoon & Sohn \\
\hline
\end{tabular}

Tab. 1: Gemeinsamer germanischer Wortschatz (Hufeisen, Marx, 2014: 28-29)

Nimmt man beispielsweise folgenden kurzen Textausschnitt auf Schwedisch, wird vieles auf einmal erkannt bzw. entschlüsselt (Namen: Alfred Bernhard Nobel, Daten: 21 oktober 1833, 10 december 1896, Ortsnamen: Stockhol, San Remo, Italien, zahlreiche internationale Wörter sowie Wörter germanischer Herkunft: svensk, kemist, dynamiten, industrialist, ingenjörn, testamente, död, berömt oder Teile von naturens, krafter, stiftare (Hufeisen, Marx, 2014: 11):

Alfred Bernhard Nobel, född 21 oktober 1833 i Stockholm, död 10 december 1896 i San Remo, Italien var en svensk kemist, uppfinnare av dynamiten, industrialist, donator, ingenjör och stiftareav Nobelpriset.

Alfred Nobel var mycket begåvad, hade stor arbetsförmåga och fast tro på mänsklighetens utveckling genom fredlig kamp med naturens krafter. Hans uppfinningar banade $n z$ väg på flera områden. Hans testamente har gjort Sverige berömt $i$ hela världen.

Für das erste Sieb sind sehr gut kurze authentische Texte aus dem Internet geeignet. Aufgrund des Textmusterwissens sowie anhand der Zahlen (Uhrzeiten, Preisen) lässt sich im folgenden Text aus der Internetseite des Van-Gogh-Museums in Amsterdam viel verstehen. Auf der Wortschatzebene verhelfen die Monatsnamen (mei, september, januari), Wochentagsnamen (vrijdag), internationale Wörter und 
Wörter germanischen Ursprungs (gratis, parkeren, bussen, adres, jaar etc.) zum Textverstehen.

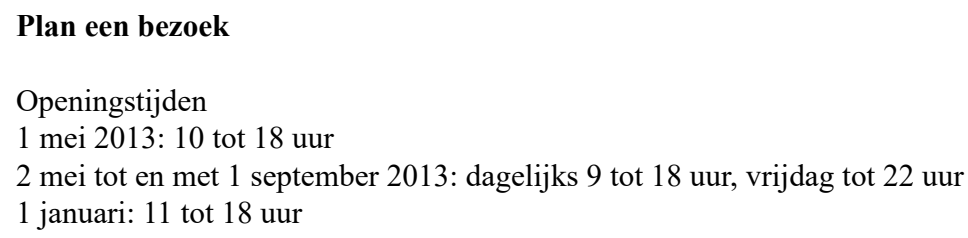

Abb. 1: Erprobung des Verstehens eines niederländischen Textes (Perge, 2014: 270)

Im zweiten Sieb wird mit Funktionswörtern gearbeitet. Sie fungieren als Textvebindungselemente und spielen deshalb beim Textverstehen eine wichtige Rolle, indem sie den so genannten roten Faden des Textes sichern (Perge, 2014: 271). Dazu gehören Pronomina, Konjunktionen, Artikelwörter, Präpositionen etc.

\begin{tabular}{|c|c|c|c|c|c|c|}
\hline Dt. & Engl. & Fries. & Nl. & Dän. & Norw. & Schw. \\
\hline ich & I & ik & ik & jeg & jeg & jag \\
\hline mir/mich & me & my & me, mij & mig & meg & mig \\
\hline
\end{tabular}

Tab. 2: Personalpronomen in der ersten Person in germanischen Sprachen (Perge, 2014: 271)

Funktionswörter erfüllen Verweisfunktionen in Texten und werden als Mittel der Kohäsion betrachtet. Um den Sinn des Textes durch diese Verweise zu verstehen, wird von Rezipienten viel Aufmerksamkeit in Bezug auf den Kontext verlangt. Im Textausschnitt Alfred Bernhard Nobel sind folgende Funktionswörter zu finden: die Präposition $i$ (in), $a v$ (von), genom (in, durch, bei), på (auf, für), med (mit); das Possessivpronomen hans (sein); die Konjunktion och (und), die Partikel mycket (viel, sehr), die Hilfsverben var (war) und har (hat) (Hufeisen, Marx, 2014: 14-15).

Das dritte Sieb „Laut- und Graphementsprechungen“ verhilft bei einer schnelleren Orienierung im Text. Viele Lexeme haben verschiedene Entwicklungsprozesse durchlaufen, deswegen werden sie nicht sofort erkannt. Als Beispiel in dem oben erwähnten Text Alfred Nobel kann man das schwedische begåaad (dt. begab $t$ ) anführen. Hier enthält das deutsche Wort ein $\langle\mathrm{b}\rangle$, wobei das schwedisch ein $\langle\mathrm{v}>$ beinhaltet (Hufeisen, Marx, 2014: 12). 
Um Lautentsprechungen erkennen zu können, sind sprachgeschichtliche Kenntnisse von Bedeutung. Die vorliegende Tabelle zeigt ein paar konsonantische Lautentsprechungen in germanischen Sprachen (Perge, 2015: 273; Oleschko, 2011: 9):

\begin{tabular}{|c|c|c|}
\hline Dän./Engl./Fries./Isl./N1./Norw./Schw. & Deutsch & Sonderfälle \\
\hline $\mathrm{v} / \mathrm{f}$ (isl. $y$ fir) & $\mathrm{b}(\ddot{u} b e r)$ & Außer am Wortanfang \\
\hline t (eng. out, eat) & s/ss/ß (aus, essen $)$ & Nach Vokal, Dän. $d$ \\
\hline $\mathrm{d}(\mathrm{nl}$. deur) & $\mathrm{t}($ Tür $)$ & $\begin{array}{l}\text { Zwischen Vokalen kann } d \\
\text { wegfallen; Engl. auch } t h \text {. }\end{array}$ \\
\hline k (dän. seks, engl. make) & ch (sechs, machen) & $\begin{array}{l}\text { Außer am Wortsanfang; } \\
\text { Dän. nach Vokal } g\end{array}$ \\
\hline p/pp (engl. ship, isl. skip; engl. help) & f/ff/pf (Schiff, Hilfe) & Am Wortanfang immer $p f$ \\
\hline
\end{tabular}

Tab. 3: Konsonantische Lautentsprechungen in germanischen Sprachen

Das vierte Sieb hilft verwandte Wörter zu erkennen. Dies betrifft die Rechtschreibung und die Aussprache, weil die Graphem-Phonem-Korrespondenzen eine wichtige Rolle beim Erwerb rezeptiver Kompetenz spielen. Bezüglich der Graphemen in germanischen Sprachen gibt es kaum Problemfälle. Außer dem Jiddischen, der einzigen germanischen Sprache, wird das lateinische Alphabet verwendet. Dennoch gibt es Unterschiede in den Lautwerten einzelner Buchstaben, d.h. die LautBuchstabenbeziehung ist in germanischen Sprachen nicht einheitlich. Als Brückensprache kann hier Deutsch gelten, denn in Bezug auf das Verhältnis zwischen Graphie und Aussprache gilt das Englische als eine der schwierigsten germanischen Sprachen.

\begin{tabular}{|c|c|c|}
\hline & Deutsch & Niederländisch \\
\hline graphisch & Kind & heb \\
\hline phonisch & {$[$ kint $]$} & {$[$ he:p] } \\
\hline
\end{tabular}

Tab. 4: Auslautverhärtung im Deutschen und Niederländischen (Perge, 2015: 274)

Das fünfte Sieb zielt auf die Herausarbeitung von Gemeinsamkeiten auf der Satzebene ab. Hier handelt es sich darum, dass der Satzbau in germanischen Sprachen viel Ähnliches aufweist. Somit ist es in einer unbekannten Sprache relativ schnell zu erkennen, was im Satz ein Subjekt, Objekt oder Prädikat ist (Hufeisen, Marx, 2014: 16).

Es ist bekannt, dass allen germanischen Sprachen die Zweitstellung des Verbs im Aussagesatz gemeinsam ist. Zu erwähnen sind aber auch Strukturen, die sich nicht allen germanischen Sprachen ähneln. Die im Deutschen, Niederländischen und Friesischen vorhandenen Satzklammern fehlen beispielsweise in den übrigen germanischen Sprachen. Diese Struktur verhilft vor allem bei der Steuerung der Aufmerksamkeit, weil es wichtig ist, das Prädikat an der richtigen Stelle zu finden. 
Vergleichen wir folgende Beispiele (mit und ohne Satzklammer):

Deutsch: Maria hat ein Bier getrunken.

Niederländisch: Maria heft een biertje gedronken.

Englisch: Mary has drunk a beer.

Schwedisch: Maria har druckit en öl.

Mit dem sechsten Sieb werden morphosyntaktische Kategorien erschlossen. Dazu gehören Konjugation, Komparation, Pluralbildung, Artikelgebrauch etc. Es sind viele Übereinstimmungen vorhanden, die aber infolge sprachgeschichtlicher Entwicklungen nicht auf Anhieb erkennbar sind. Nehmen wir z.B. den Tempusgebrauch, der auch zu der morphosyntaktischen Ebene gehört. In germanischen Sprachen hat das Prinzip der Bildung des Präteritums viele Übereinstimmungen, z.B. dt. er trank, engl. he drank, nl. hij dronk, dän. han drank, norw. han drakk.

Im Text Alfred Bernhard Nobel können der enklitische (am Nomen angefügt) Artikelgebrauch (z.B. dynamiten, Nobelpriset oder världen) und die Pluralbildung im Lexem uppfinningar als morphosyntaktische Kategorien veranschaulicht werden.

Im letzten Sieb erfolgt die Analyse der Affixe, die dem Leser dazu verhelfen, größere Teile des Wortschatzes zu verstehen. Dabei können die häufigsten Wortbildungsbausteine der jeweiligen germanischen Sprache erschlossen werden, weil zahlreiche Affixe Äquivalente in einer anderen germanischen Sprache haben:

\begin{tabular}{|c|c|c|c|}
\hline Deutsch & Niederländisch & Dänisch & Schwedisch \\
\hline beschließen & besluiten & beslutte & besluta \\
\hline erfahren & ervaren & erfare & erfara \\
\hline
\end{tabular}

Tab. 5: Präfixe in germanischen Sprachen (Perge, 2015: 276)

Aus dem Text Alfred Bernhard Nobel können folgende Elemente bzw. Äquivalente erwähnt werden: das Präfix ut- (dt. ent-) und das Suffix -ing (dt. -ung) im Wort utveckling, die sofort erkannt werden können „Entvecklung“ (Hufeisen, Marx, 2014: 14). Die folgende Tabelle präsentiert einige Affixe und ihre Entsprechungen:

\begin{tabular}{|c|c|c|c|c|}
\hline Wortart & Deutsch & Luxemburgisch & Afrikaans & Dänisch \\
\hline Nomen & $\begin{array}{c}\text {-schaft } \\
\text { Freundschaft }\end{array}$ & $\begin{array}{c}\text {-schaft } \\
\text { Frëndschaft }\end{array}$ & $\begin{array}{c}\text {-skap } \\
\text { vriendskap }\end{array}$ & $\begin{array}{c}\text {-skab } \\
\text { venskab }\end{array}$ \\
\hline Adjektive & $\begin{array}{c}\text { un-; -lich } \\
\text { unmöglich }\end{array}$ & $\begin{array}{c}\text { on-; -lech } \\
\text { onméglech }\end{array}$ & $\begin{array}{c}\text { on-; -lik } \\
\text { unmakelik }\end{array}$ & $\begin{array}{c}\text { u-; -lig } \\
\text { unmulig }\end{array}$ \\
\hline Verben & $\begin{array}{c}\text { vor- } \\
\text { vorbereiten }\end{array}$ & $\begin{array}{c}\text { vor- } \\
\text { vibereden }\end{array}$ & $\begin{array}{c}\text { voor- } \\
\text { voorberei }\end{array}$ & $\begin{array}{c}\text { for- } \\
\text { forberede }\end{array}$ \\
\hline
\end{tabular}

Tab. 6: Affixe in germanischen Sprachen (Olescho, 2011: 14) 


\section{Relevanz für Fremdsprachenunterricht}

„Wer fremde Sprachen nicht kennt, weiß nichts von seiner eigenen“ stellte vor mehr als 180 Jahren der berühmte deutsche Schriftsteller Johann Wolfgang von Goethe in seinem Werk Maximen und Reflexionen (1881) fest. Aus diesem Einsatz für Mehrsprachigkeit lässt sich leicht schließen, dass die Beherrschung einer Fremdsprache heute nichts von seiner Aktualität verloren hat. Das Fremdsprachenlernen gehört im gegenwärtigen Kontext der Globalisierung zur Allgemeinbildung, bereichert unsere innere Welt und ist auf dem Arbeitsmarkt von unbestreitbarem Vorteil.

Im Bereich der Mehrsprachigkeitsdidaktik werden immer wieder neue Methoden für die bessere Vermittlung der Fremdsprachen entwickelt. Obwohl Mehrsprachgkeit ein wichtiger Bestandteil europäischer Bildungsziele ist, findet man leider selten in den Lehrwerken für Fremdsprachen Elemente der Mehrsprachigkeitsdidaktik. Trotzdem bin ich der Meinung, dass Ansätze der Mehrsprachigkeits- bzw. Tertiärsprachendidaktik eine bedeutende Ressource im Fremdsprachenunterricht sein können. Mittelerweile gibt es vonseiten der Fremdsprachendidaktik mehrere Konzepte zur Sensibilisierung der Mehrsprachigkeit im Unterricht. Dies bestätigen die schon erwähnten unterrichtspraktischen Interkomprehensionsmodelle wie $E \mathbf{~ - ~}$ roComRom, EuroComGerm und EuroComSlav.

Darüber hinaus gibt es in der ganzen Welt mehrere Bildungsinstitutionen, vor allem allgemeinbildende Schulen, in denen Sachfächer in einer Fremdsprache unterrichtet werden. Der Begriff Content and Language Integrated Learning (CLIL) hat sich für dieses didaktische Konzept durchgesetzt und wird in vielen Ländern erfolgreich durchgeführt. Daraus lässt sich behaupten, dass die Konzepte der Mehrsprachigkeitsdidaktik immer mehr ihre Anwendung im Unterricht finden.

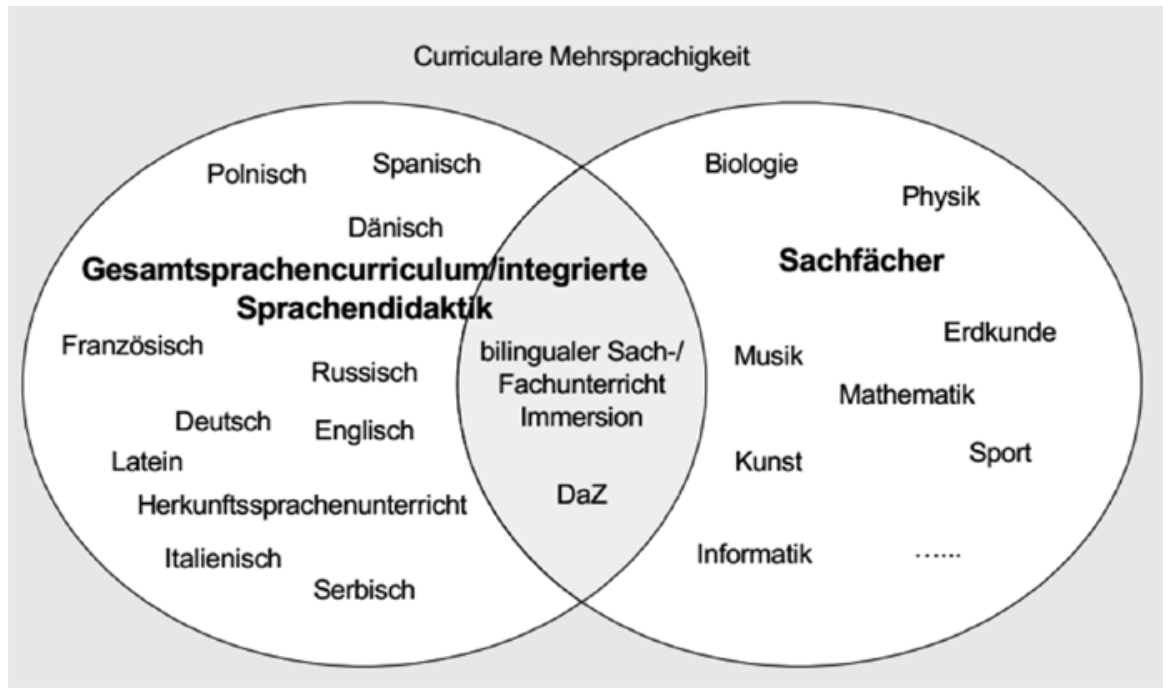

Abb. 2: Gesamtsprachencurriculum (Hufeisen, 2012: 14) 
Darüber hinaus geriet in den vergangenen beiden Jahrzehnten die Tertiärsprachenforschung in den Fokus der rezeptiven Mehrsprachigkeit. Das ist beispielsweise ein solches Projekt, wie Deutsch als Fremdsprache nach Englisch (DaFnE). Es untersucht die besondere Konstellation, wenn Deutsch nach der Fremdsprache Englisch erlernt wird. Der Grund für die Entstehung dieses Projekts war die Erkenntnis, dass ausländische Deutschlerner weltweit hauptsächlich Englisch als erste Fremdsprache lernen oder gelernt haben. Diese Sprachlernerfahrung kann man im Deutschunterricht ausnutzen, indem Vergleiche mit dem Englischen gezogen werden und die Ähnlichkeiten sowie Unterschiede bewusst gemacht werden. Dabei ist der Bezug des Deutschen zum Englischen insbesondere dann effektiv, wenn die Muttersprache der Lerner wenige Gemeinsamkeiten mit dem Deutschen aufweist. In diesem Fall kann die erste Fremdsprache Englisch als Brücke betrachtet werden. Das Konzept EuroCom bzw. EuroComGerm hat viele Anknüpfungspunkte zu der DaFnE-Konstellation, weil es sich beim Englischen und Deutschen um germanische Sprachen handelt.

Alle Ähnlichkeiten zwischen Sprachen sind große Hilfen im Fremdsprachenerwerb. Jeder Lerner sucht (bewusst oder unbewusst) nach den sogenannten „Transferbrücken“: „Welche Elemente, Einheiten, Strukturen der Muttersprache (L1) und der ersten Fremdsprache (L2) können mit den vergleichbaren Elementen, Einheiten und Strukturen der Tertiärsprache (L3) in Beziehung gesetzt und verbunden werden?" (Neuner, 2003: 25). Beim Bau dieser Transferbrücken können u.a. Internationalismen, Lehnwörter sowie ähnliche grammatische und syntaktische Strukturen eine wichtige Rolle spielen. Hier sind einige Beispiele aus den gegenwärtigen Lehrwerken, die diese Ähnlichkeiten berücksichtigen. Dabei handelt es sich um Aufgaben, innerhalb derer sich die Gemeinsamkeiten vorwiegend auf die Phänomene stützen, die im ersten Sieb des EuroComGerm-Projekts ihre Beachtung finden:

a) die internationale Lexik bzw. Internationalismen (z.B. dt. Organisation, Meter, Taxi, Musik, Struktur usw.), ,[...] die zwar in Schreibung und Lautung dem Sprachsystem der jeweiligen Sprache angepasst wurden, deren Bedeutung aber „über die Sprachen hinweg " gleich geblieben ist" (Kursiša, Neuner, 2006: 4).

b) der Übernahmen aus dem Englischen (Anglizismen als Fremd- und Lehnwörter, z.B. dt. Jeans, PC, Internet, Party, Interview etc.), die ihre Verbreitung vor allem in solchen Bereichen wie Jugendkultur, Sport, Technik, Mode, Medien oder Gastronomie fanden.

c) der gemeinsame germanische Ursprung (z. B. Wörter aus solchen Themenbereichen wie Speisen und Getränke, Wetter und Natur, Tiere und Pflanzen, Freizeit und Sport, Mode und Kleidung, Verwandtschaftsbezeichnungen, Körperteile, Wochentage etc.), z.B.: 
die Mutter · der Onkel · der Cousin · die Kinder · der Großvater die Nichte · die Cousine - die Enkelkinder · der Neffe · die Tante der Bruder - die Eltern - die Frau · der Sohn - die Großmutter die Großeltern · die Schwester · die Tochter · des vater - der Mann

die Mutter - der Vater husband - wife - father mother - sister - brother daughter $\cdot$ son - children parents - uncle - aunt grandfather $\cdot$ grandmother grandparents - grandchildren niece - nephew - cousin

Abb. 3: Beispielübung aus „Deutsch ist easy!“ (Kursiša, Neuner, 2006: 34)

Die germanische Sprachenvielfalt kann ohne Zweifel im DaF-Unterricht zum Thema gemacht werden, weil die meisten Lernenden mindestens eine germanische Sprache - das Englische - in ihrem Sprachrepertoire haben. Die folgende Tabelle verdeutlicht, dass eine Auseinandersetzung mit dem Sprachenvergleich schon im Anfängerunterricht möglich ist. Wenn wir nur einen kurzen Blick auf die BegrüBungsformel „Guten Morgen“ werfen, ist die Verwandschaft der Sprachen offensichtlich. Dies lässt sich besonders im Kontrast zu den nicht verwandten Sprachen erkennen:

\begin{tabular}{|c|c|c|c|}
\hline \multicolumn{2}{|c|}{ germanische Sprachen } & \multicolumn{2}{|c|}{ andere Sprachen } \\
\hline Deutsch & Guten Morgen & Türkisch & Günaydın \\
\hline Niederdeutsch & Moin & Finnisch & Hyväähuomenta \\
\hline Jiddisch & \begin{tabular}{|l} 
גוטן מארָרגן \\
(Gutn murgn)
\end{tabular} & Griechisch & 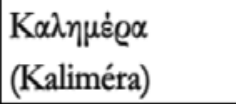 \\
\hline Luxemburgisch & Moien & Polnisch & Dzień dobry \\
\hline Niederländisch & Goede morgen & Russisch & $\begin{array}{l}\text { Aoбpoe yrpo } \\
\text { (Dobroe utro) }\end{array}$ \\
\hline Afrikaans & Goeiemôre & Albanisch & Mirëmëngjes \\
\hline Englisch & Good morning & Litauisch & Labas rytas \\
\hline Dänisch & God morgen & Walisisch & Bore da \\
\hline
\end{tabular}

Tab. 7: Guten Morgen in germanischen und anderen Sprachen (Oleschko, Olfert, 2014: 53)

Durch das breite gemeinsame Sprachpotenzial, vor allem im Wortschatzbereich, kann im Anfangsunterricht im Verstehensbereich schnell und effizient ein relativ großer Grundwortschatz des Deutschen aufgebaut werden. In den heutigen Lehrwerken geht es meistens um Aufgaben, bei denen der Wortschatz aus dem thematisierten Wortfeld gegenübergestellt und verglichen wird. 


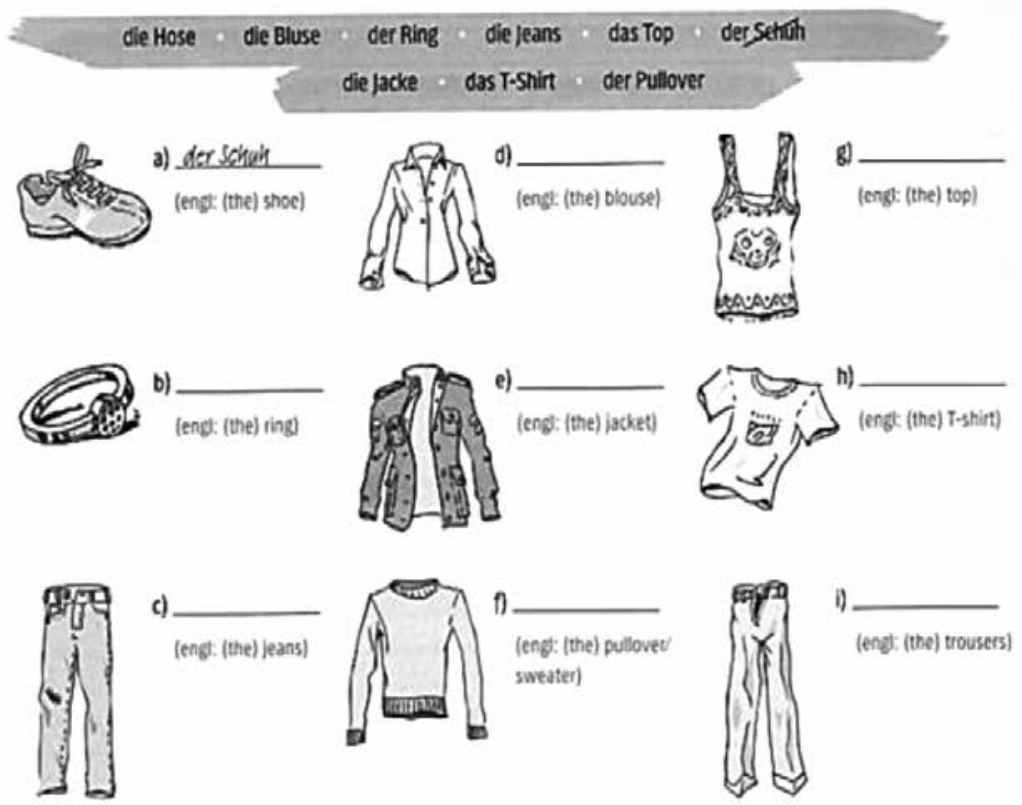

Abb. 4: Beispielübungen (Deutsch.com, Arbeitsbuch, S. 14)

Es führt dazu, dass Lernende die Ähnlicheiten in den Wortschätzen der beiden Sprachen erkennen und sich die deutschen Vokabeln schneller merken. Bei solchen Aufgaben kann auch auf die Unterschiede in der Schreibung und Aussprache hingewiesen werden (vgl. Vicente, Pilypaityte, 2015: 54). Eine ähnliche Vorgehensweise findet ihren Platz auch in den „sieben Sieben“ des EuroCom Germ-Projekts (z.B. Sieb 3 „Laut- und Graphementsprechungen“ und Sieb 4 „Rechtschreibung und Aussprache"):

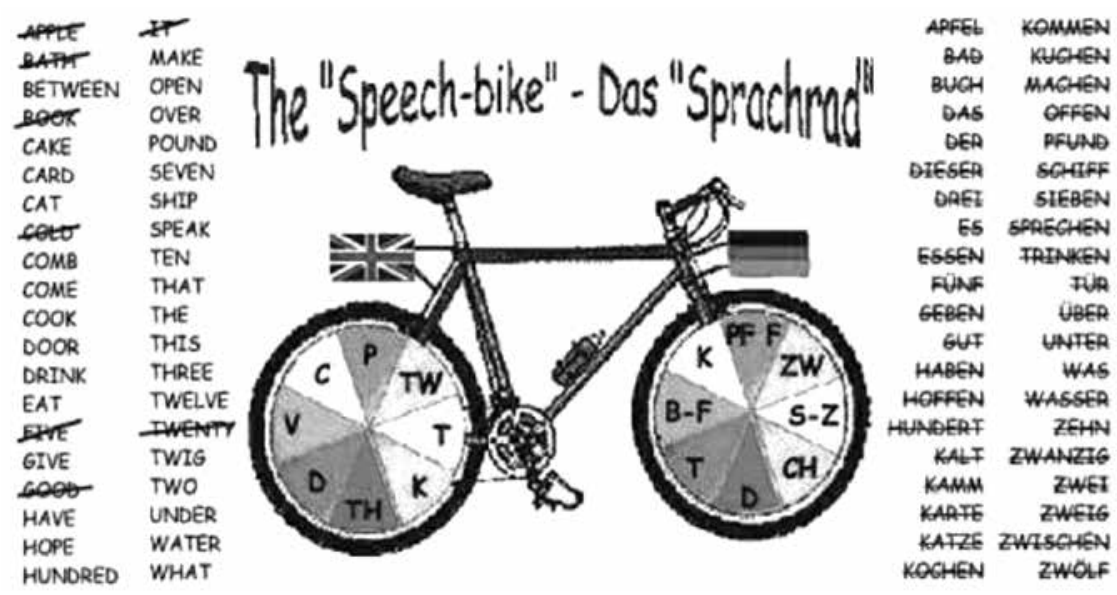

Abb. 5: Das Sprachrad für Orthographie - Deutsch und Englisch 
Die sog. ,fremde Welt" soll im Fremdsprachenunterricht vor allem mediengebunden präsentiert werden, z.B. in Lese- und Hörtexten, Bildern, Videos, Filmen etc. (vgl. Kursiša, Neuner, 2006: 6). Auch die Arbeit mit Lesetexten nimmt von Anfang an einen wichtigen Platz im Tertiärsprachenunterricht ein. Die sog. L3-Textdidaktik bezieht sich auf die Arbeit mit synthetischen Texten:

a) Text zu einem Wortfeld erarbeiten: Die Aufgaben sollen die Lernenden zum selbständigen Arbeiten anleiten, z.B. sie sollen einen deutschen Text mit vielen Internationalismen (Fremd- Lehnwörtern) global verstehen und erkennen können, dass nicht jedes einzelne Wort eines Textes übersetzt werden muss, um seinen Sinn zu erfassen. Hier ist ein Beispieltext aus dem Lehrwerk studio d, der darauf hinzielt, einen Text zu Wortfeldern (Kaffee, Geografie, andere) zu erarbeiten:

13 Café International. Welche Wörter verstehen Sie? Notieren Sie.

Das Kaffeetrinken ist eine arabische Tradition. Die Türken haben Mokka international populär gemacht. In Europa hat Österreich eine lange Kaffeehaustradition und viele Kaffeevariationen.

Heute ist Kaffeetrinken „in“. Caffè Latte, Espresso und Cappuccino heißen die TopFavoriten in Hongkong, New York, Berlin und St. Petersburg. Café-Ketten wie Starbucks, Segafredo und Coffee Bean sind so international wie McDonalds. Cafés sind ideal für die Kommunikation und für Kontakte.

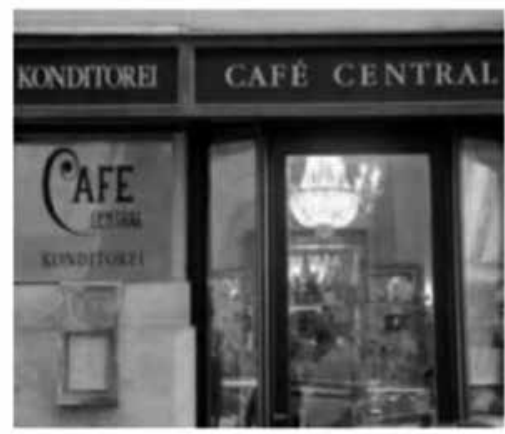

\begin{tabular}{|c|c|c|}
\hline Kaffee & Geografie & andere \\
\hline & & ideal \\
\hline
\end{tabular}

Abb. 6: Beispieltext aus dem Lehrwerk studio $d$, Kurs- und Übungsbuch, S. 28

b) (Englisch-deutsche) Paralleltexte: Mit Hilfe der sog. „Paralleltexte“, die vor allem viele Internationalismen enthalten, kann der deutsche Text selbständig durch paralleles Lesen in seiner globalen Bedeutung erfasst werden. Durch die vergleichende Analyse von derartigen synthetischen „Paralleltexten“ können die Lernenden dazu angeregt werden, sich die Sprachsysteme im Bereich von Wortschatz und Grammatik selbständig zu erschließen. 
Freundinnen

Nadine lebt in Wien, Silvia in Hamburg und Esther in Luzern. Sie telefonieren oft, senden einander Fotos und schreiben E-Mails oder Postkarten. Und immer am ersten Wochenende im Juni machen sie einen Kurztrip und kommen für drei Tage zusammen. Dieses Jahr kommen sie nach Frankfurt.

Vor acht jahren waren alle drei in China und lernten dort an einer Sprachschule Chinesisch. Sie wurden gute Freundinnen. Dann waren sie wieder zu Hause und blieben in Kontakt. Bald begannen sie mit der Tradition, einmal im Jahr, am ersten Juniwochenende, zusammenzukommen. Für mein Interview warte ich im Hotel in Frankfurt auf Nadine, Silvia und Esther. Ich will sie nach den Hobbys fragen, durch die sie nach China gekommen sind und sich dort ..

\section{Friends}

Nadine lives in Vienna, Silvia in Hamburg and Esther in Berne. They phone often, send each other photos and write e-mails or postcards. And every year at the first weekend in June they go on a trip and come together for three days. This year they come to Frankfurt. Eight years ago all three of them were in China and learned Chinese at a language-school there. They became good friends.

Then they were home again and kept in contact. Soon they began a tradition: once a year - at the first weekend in June - they would come together. I am waiting for Nadine, Silvia and Esther in a hotel in Frankfurt. I want to get an interview and ask them about their hobbys...

Abb. 7: Beispielt für einen Paralleltext (Kursiša, Neuner, 2006: 77)

Das wichtigste Ziel der Entwicklung des Leseverstehens ist auch der kompetente Umgang mit authentischen fremdsprachlichen Texten, die vor allem im Internet zu finden sind. Für den erfolgreichen Lernprozess erhalten sie einen hohen Anteil an Wörtern aus dem gemeinsamen germanischen Wortschatz, an Internationalismen und an Lehnwörtern aus dem Englischen. So können sie in ihrer globalen Bedeutung relativ leicht erschlossen werden. Dabei können die „sieben Siebe“ als Texterlschließungsstrategien eine große Hilfe anbieten.

\section{Fazit}

Im Bereich der Inter- und Eurokomprehensionsforschung gibt es Ansätze, die auf der Grundlage des Kontrastierens von Sprachen erprobt werden und auf die rezeptive Mehrsprachigkeit abzielen. Solche Projekte wie EuroComGerm und die „sieben Siebe“, DaFnE oder CLIL verhelfen den Lernenden, sich im Unterricht anderen Sprachen zu nähern, sie zu erkunden und zu entdecken. Dabei bieten diese Methoden den Lernenden an, durch Kontrastivität eine neue Fremdsprache anders kennen zu lernen.

Die Strategien des Sprachvergleichs sind für mehrsprachige Lernende eine der Möglichkeiten, mit Unbekanntem umzugehen. Der im vorliegenden Beitrag vorgestellte germanische Interkomprehensionsansatz ist eine dieser Möglichkeiten. Er ist in seiner Komplexität nicht für jede Zielgruppe geeignet, dennoch kann er im Fremdsprachenunterricht einen wichtigen Platz einnehmen. Bei Bedarf können nicht alle Siebe berücksichtigt und nur teilweise im Lernprozess angesetzt werden. Der Vorteil des Projekts besteht darin, dass Lernende eine Systematik kennen lernen und Regeln entdecken, die ihnen für das Erlernen einer weiteren Fremdsprache nützen können. Dadurch wird eine mehrsprachige Kompetenz erreicht sowie die individuelle Sprachlernbewusstheit berücksichtigt. 
Damit der interkomprehensive Ansatz im Unterricht gelingt, muss die Lehrkraft dazu bereit und fähig sein, Sprachvergleiche in die Lehrmaterialien zu integrieren. Unterschiedliche mehrsprachigkeitsfördernde Aufgaben- und Übungsformen, die auf den Prinzipien der Mehrsprachigkeitsdidaktik bzw. Tertiärsprachendidaktik basieren, finden leider selten Eingang in die Lehrbücher. Die Ressource der Mehrsprachigkeit soll aber im Lehrprozess genutzt und angemessen eingesetzt werden, denn alle Ähnlichkeiten zwischen Sprachen, die dem Lerner den Erwerb einer weiteren Fremdsprache erleichtern, sind willkommene Hilfen im Fremdsprachenunterricht. Sie ermöglichen nicht nur die Orientierung in einer fremdsprachigen Umgebung, sondern sind beim Erlernen einer Fremdsprache als Gedächtnishelfer sehr hilfreich.

\section{Literaturverzeichnis}

1. Bär, M. (2012). Einführung in die (romanische) Mehrsprachigkeitsdidaktik. In: Hildenbrand, E.; Martin, H.; Vences, U. (Hrsg.): Mehr Sprache(n) durch Mehrsprachigkeit. Erfahrungen aus Lehrerbildung und Unterricht. Berlin: Verlag Walter Frey, S. 7-22.

2. Cosentino, G. (2016). Mehrsprachige Kompetenz: Welche Anforderungen an den DaZ-Unterricht? In: https://www.academia.edu/24553923/Mehrsprachige_Kompetenz_Welche_Anforderungen_an_den_DaZ-Unterricht [abgerufen am 07.06.2019]

3. Neuner, G. et.al. (Hrsg.). (2010). Deutsch.com. Lehr- und Arbeitsbuch. Ismaning: Max Hueber Verlag.

4. Goethe von, J. W. (1981). Maximen und Reflexionen. München: Beck.

5. Hinrichs, U. (2009). Geschichte, Stand und Perspektiven der Eurolinguistik. In: Hinrichs, U.; Reiter, N.; Tornow, S. (Hrsg.). Eurolinguistik. Entwicklungen und Perspektiven. Eurolinguistische Arbeiten. Akten der Internationalen Tagung vom 30.9.-2.10.2007 in Leipzig. Wiesbaden: Harrassowitz, S. 1-49.

6. Hufeisen, B.; Marx N. (Hrsg.). (2007). EuroComGerm-Die sieben Siebe: Germanische Sprachen lesen lernen. Herzogenrath: Shaker Verlag.

7. Hufeisen, B.; Marx N. (Hrsg.). (2014). EuroComGerm-Die sieben Siebe: Germanische Sprachen lesen lernen. 2. Auflage. Aachen: Shaker Verlag.

8. HufeisenB.(2012).MutzurMehrsprachigkeit.Neuesaus TheorieundPraxis. In: https://www.ecml.at/Portals/1/mtp4/plurcur/documents/2012\%20Hufeisen\%20 Vortrag\%20Dornbirn.pdf [abgerufen am 07.06.2019]

9. Klein, H. G.; Stegmann, T. D. (2001). EuroComRom - Die sieben Siebe. Romanische Sprachen sofort lesen können. 3. Aufl., Aachen.

10. Kommission der Europäischen Gemeinschaften (2005). Mitteilung der Kommission an den Rat, das Europäische Parlament, den Europäischen Wirtschafts- und Sozialausschuss und den Ausschuss der Regionen. Eine neue Rahmenstrategie für Mehrsprachigkeit. In: https://eur-lex.europa.eu/legal-content/DE/ 
TXT/?uri=celex:52005DC0596 [abgerufen am 07.06.2019]

11. Kursiša, A.; Neuner G. (2006). Deutsch ist easy! Lehrerhandreichungen und Kopiervorlagen „Deutsch nach Englisch“ für den Anfangsunterricht. Ismaning: Max Hueber Verlag.

12. Neuner, G. (2003). Mehrsprachigkeitskonzept und Tertiärsprachdidaktik. In: Hufeisen, B.; Neuner, G. (Hrsg.). Mehrsprachigkeitskozept - Tertiärsprachenlernen - Deutsch nach Englisch. Strasbourg: Council of Europe Publishing, S. 13-34.

13. Neuner, G. (2004). Deutsch als Herausforderung. Fremdsprachenunterricht und Literatur in Forschung und Lehre. Festschrift für Rainer Kussler. Stellenbosch: Sun Press, S. 37-54.

14. Oleschko, S. (2011). Interkomprehension am Beispiel der germanischen Sprachen. In: https://www.uni-due.de/imperia/md/content/prodaz/interkomprehension20110412.pdf [abgerufen am 17.06.2019]

15. Oleschko, S., Olfert, H. (2014). Interkomprehensionsorientierung zur Anerkennung sprachlicher Vielfalt. In: Ferraresi, G., Liebner, S. (Hrsg.). SprachBrückenBauen. 40. Jahrestagung des Fachverbandes Deutsch als Fremd- und Zweitsprache an der Universität Bamberg. Bd. 92. Universitätsverlag Göttingen, S. 47-60.

16. Perge, G. (2014). Intercomprehension. Gemeinsamkeiten verwandter Sprachen beim Erwerb der fremdsprachlichen Lesefähigkeiten nutzen lernen. In: Feld-Knapp, I. (Hrsg.). Mehrsprachigkeit. Bd. 2. Cathedra Magistrorum-Lehrerforschung. Budapest: Eötvös-József-Collegium, S. 267-310.

17. Funk, H. et.al. (Hrsg.). (2006). Studio d. Lehr-und Arbeitsbuch. Berlin: Cornelsen.

18. Surkamp, C. (Hrsg.). (2010). Metzler Lexikon Fremdsprachendidaktik. Stuttgart: Metzler.

19. Zybatow, L. (2002). Die slavistische Intercomprehensionsforschung und Eurocomslav. In: Kischel, G. (Hrsg.). EUROCOM. Mehrsprachiges Europa durch Eurocomprehension in Sprachfamilien. Tagungsband des Internationalen Fachkongresses im Europäischen Jahr der Sprachen. Hagen: BasseDruck, S. 357-371.

20. Vicente, S.; Pilypaitytė L. (2015). Lehrsprachigkeitsdidaktik in Lehrmaterialien. In: Fremdsprache Deutsch 50, S. 52-58. 


\title{
MULTILINGUALISM AS A RESOURCE IN FOREIGN LANGUAGE TEACHING
}

\author{
Dr. Diana Babušytè \\ Mykolas Romeris University
}

Multilingualism is an important element in the language policy of the European Union (EU). The European Commission encourages the EU citizens to be multilingual and promotes learning of at least two foreign languages in addition to the mother tongue. In this context, foreign language teaching and learning are of great importance nowadays.

The didactics of multilingualism is a relatively new term in the European and worldwide foreign language didactics. It aims to encourage the learners' interest in foreign language learning and distance from the traditional monolingual concept in foreign language teaching. This paper stresses the promotion of individual multilingualism in foreign language teaching. It presents the main principles of intercomprehension and the EuroCom projects which concentrate on the similarities among related languages and aim to promote receptive multilingualism. In addition, the methods of "seven sieves" of the EuroComGerm project and DaFnE (Deutsch als Fremdsprache nach Englisch) are presented as learning strategies based on the contrastive comparison of Germanic languages in reading skills. The advantage of the methods is that multilingual learners discover the rules and strategies that can help them learn a foreign language.

Different forms of exercises promoting multilingualism and the ones based on the principles of multilingualism didactics and tertiary didactics rarely find their way into textbooks. However, the multilingual resources should be used and adequately addressed in the teaching process. All similarities among languages, which make it easier for the learner to acquire another foreign language, are welcome aids in foreign language teaching. They not only provide orientation in a foreign-language environment but also are very helpful in learning a foreign language as a reminder. On the basis of examples, the paper explores the question of how the research on multilingualism didactics can be used effectively as a resource in foreign language teaching and how it can contribute to the promotion of multilingual competence. 


\section{AUTORIAUS LYDRAŠTIS}

Autoriaus vardas, pavardė: Diana Babušytė

Mokslo laipsnis ir vardas: humanitarinių mokslų daktarè

Darbo vieta ir pareigos: Mykolo Romerio universiteto profesore

Autoriaus mokslinių interesų sritys: dabartinè vokiečių kalba, kontrastyvinė lingvistika, eurolingvistika, kalbų tipologija, svetimujų kalbų dėstymo didaktika.

Telefonas ir el. pašto adresas: +370 64590 727; diana.babusyte@mruni.eu

\section{AUTHOR'S COVER LETTER}

Author's name and surname: Diana Babušytė

Academic degree and name: Doctor of Arts

Workplace and position: Professor, Doctor, Mykolas Romeris University

Author's research interests: modern German, contrastive linguistics, Eurolinguistics, linguistic typology, foreign language teaching methods

Telephone and e-mail address: +37064590 727; diana.babusyte@mruni.eu 